\title{
AVALIAÇÃO DA SÉRIE HISTÓRICA DOS NASCIDOS VIVOS EM UNIDADE TERCIÁRIA DE PERNAMBUCO - 1991 A 2000
}

\author{
Suely A. Vidal*; Bertoldo K. G. de Arruda, lygia C. Vanderlel, Paulo G. Frias \\ Trabalho realizado no Instituto Materno Infantil de Pernambuco (IMIP) - Diretoria de Pesquisa - \\ Grupo Avaliação, Recife, Pernambuco
}

RESUMO - OBjeTIVO. Analisar a série temporal dos Nascidos Vivos do Instituto Materno Infantil de Pernambuco (IMIP) - Recife, segundo algumas variáveis, no periodo de julho de $199 \mid$ a dezembro de 2000 .

Métooos. Utilizou-se 0 banco de dados do Sistema de Informa. ção sobre Nascidos Vivos (Sinasc). Para análise estatística considerou-se os anos de 1993 a 2000 e foi realizado o Qui-quadrado de tendência $(p<0,05)$ no Epi Info (versão 6.0).

REsultados. Apesar da predominância de parto vaginal, peso $\geq 2500 \mathrm{~g}$, idade gestacional $\geq 37$ semanas, Apgar no $1^{\circ}$ e $5^{\circ}$ minutos entre 8 e 10, mães adultas, 4 a 6 consultas de pré-natal, encontrou-se percentuais elevados e tendência progressiva na década para: partos cesarianos $\left(29,4 \%\right.$ a $\left.35,2 \%, \chi^{2}=73,7 ; p<0,01\right)$; baixo peso $\left(15,9 \%\right.$ a $\left.22,8 \%, \chi^{2}=170,6 ; p<0,01\right)$; pré-termos $(9,7 \%$ a $23,8 \%$, $\left.\chi^{2}=503,6 ; p<0,001\right)$; mães adolescentes $\left(24,1 \%\right.$ a $28,8 \%, \chi^{2}=$ 13,3; $p<0,0 \mid$ ) além de um alto percentual de bebês com índice de Apgar menor que 3 no $1^{\circ}$ minuto de $3,4 \%$ a $5,5 \%$ e no $5^{\circ}$ minuto de $0,7 \%$ a $1,6 \%$ ).

Conclusoes. Os resultados ratificam a caracterização do IMIP como hospital de complexidade terciária e de referência para gestantes e recém-nascidos de alto risco no Estado de Pernambuco.

UnITERMOS: Avaliação em saúde. Sistemas de informação. Declaração de nascidos vivos.

\section{INTRODUÇÃO}

A implantação do Sistema de Informações sobre Nascidos Vivos (Sinasc) pelo Ministério da Saúde em 1990 ocorreu de forma gradativa nas Secretarias Estaduais de Saúde das unidades federadas ${ }^{1-5}$. Em Pernambuco, se deu inicialmente em uma única unidade hospitalar - Instituto Materno Infantil de Pernambuco (IMIP) - Recife, como projeto piloto, no segundo semestre de 1991. Porém, a Declaração de Nascidos Vivos começou a ser implantada oficialmente em Pernambuco a partir de setembro de 1992, tanto pela Secretaria de Saúde da Prefeitura do Recife quanto pela Secretaria Estadual de Saúde. Segundo documento interno da Diretoria de Informação da Secretaria Estadual de Saúde, o Sinasc alcançou 91,57\% de cobertura em 1993, e a partir daí, foi ampliado, chegando a $100 \%$ em 1999, calculado com base na taxa bruta de natalidade estimada pelo Ministério da Saúde para Pernambuco.

O Sinasc utiliza como fonte de dados a Declaração de Nascidos Vivos (DN), sendo

\footnotetext{
* Correspondência:

Rua Francisco da Cunha, 1910/102B

CEP: 51020 - 041 - Boa Viagem - Recife/PE e-mail: $\quad$ crianca@imip.org.br
}

sua emissão de responsabilidade dos serviços de saúde onde ocorreram os partos ou onde mães e recém-nascidos foram atendidos de imediato. Para os outros casos, incluindo os partos domiciliares ou em outros locais, onde não houve assistência por profissionais de saúde, é emitida pelos Cartórios de Registro Civil ou pelas Secretarias de Saúde de alguns municípios de Pernambuco, apesar dessa última forma de emissão não constar da normatização da portaria de $n^{\circ} 475$ de $31 / 08 / 2000$ da Funasa/MS 6 . O preenchimento desse documento para cada criança nascida viva passou a ter caráter obrigatório no ano de 1990, previsto no Estatuto da Criança e do Adolescente ${ }^{1-5}$.

A DN é um impresso pré-numerado em três vias e distribuído às Secretarias Estaduais de Saúde pela Fundação Nacional de Saúde. Seu fluxo varia de Estado para Estado 3 desconsiderando a portaria citada anteriormente $^{6}$, mas em geral, a primeira via (branca) é recolhida pelas Secretarias Municipais de Saúde para processamento. A segunda (amarela) fica com a família até ser levada ao cartório de registro civil para o devido registro de nascimento da criança. Em geral, o cartório retém esta via para os procedimentos legais, porém, o destino final da segunda via depende das disposições da Corregedoria de cada estado. A terceira via (rosa) deverá ser arquivada na unidade de saúde onde ocorreu o parto, no prontuário da puérpera ou da criança $^{1-8}$. A referida Portaria que regulamenta as rotinas de coleta e envio de informações para o Sinasc determina que, para os partos domiciliares, a via rosa deverá ser entregue aos familiares para ser apresentada na primeira consulta na unidade de saúde.

A partir do preenchimento deste formulário se obtém dados para a construção do perfil dos nascidos vivos em todo território nacional, tais como peso ao nascer, duração da gestação, tipo de parto, índice de Apgar e paridade e o fornecimento de informações sobre natalidade para todos os níveis do sistema de saúde. Estas informações sobre a natalidade são fundamentais para o planejamento adequado das políticas de saúde, para a avaliação de ações de saúde e para a adoção de medidas de vigilância. São também de grande utilidade para a construção de indicadores demográficos e de saúde na área materno-infanti| ${ }^{5,7}$.

O processamento dos dados e análise de consistência das DN era, inicialmente, de responsabilidade exclusiva das Secretarias Estaduais e passou progressivamente às Secretarias Municipais de Saúde, porém com supervisão do Estado. Após o processamento no município, são enviadas para as Secretarias 
Estaduais que consolidam os dados do Estado e remetem ao Ministério da Saúde para alimentar o Sistema Nacional ${ }^{1-5,8}$. A descentralização do processamento do Sinasc agilizou a disponibilidade das informações no nível local, tornando possível sua utilização imediata, embasando o planejamento para investimentos direcionados a grupo materno infantil.

Este estudo teve como objetivo avaliar a série histórica dos nascidos vivos no Instituto Materno Infantil de Pernambuco (IMIP), na década de 90, segundo algumas variáveis contidas nas Declarações de Nascidos Vivos.

\section{Métodos}

Foi realizado um estudo epidemiológico descritivo, censitário, de tendência, no Instituto Materno Infantil de Pernambuco (IMIP), que presta assistência na área materno infantil nos três níveis de complexidade. É centro de referência para o Ministério da Saúde e organismos internacionais na implantação de programas, serviços, pesquisas e treinamentos.

OIMIPé um complexo hospitalar formado pelo Hospital Geral de Pediatria, com 174 leitos e pelo Centro de Atenção à Mulher, contando com 130 leitos obstétricos, sendo 30 de cuidados intermediários, destes, dois de terapia intensiva. Dispunha, à época do período do estudo, de uma unidade neonatal com 25 berços, dos quais dois eram de UTI. Oferece atendimento ambulatorial em ginecologia, obstetrícia e pediatria, geral e especializado. A população assistida é proveniente tanto da cidade do Recife, como do interior do Estado de Pernambuco e também de outros Estados do Norte e Nordeste brasileiro. A grande maioria é de baixa renda e usuária do Sistema Único de Saúde (SUS). Ao longo do período estudado não houve ampliação na oferta de serviços pelo IMIP e não existia, até o ano de 2000, um sistema de referência estadual oficial para as gestantes de risco. Além do IMIP, há mais quatro unidades na cidade do Recife que prestam assistência às gestantes de alto risco, contudo todas possuem menor quantidade de leitos.

O número de nascidos vivos no IMIP representou aproximadamente $3 \%$ dos 160.55 | nascimentos do Estado de Pernambuco e cerca de $19 \%$ dos 27.584 dos residentes no Recife, para o ano de $1999^{9}$.

Fizeram parte deste estudo todas as

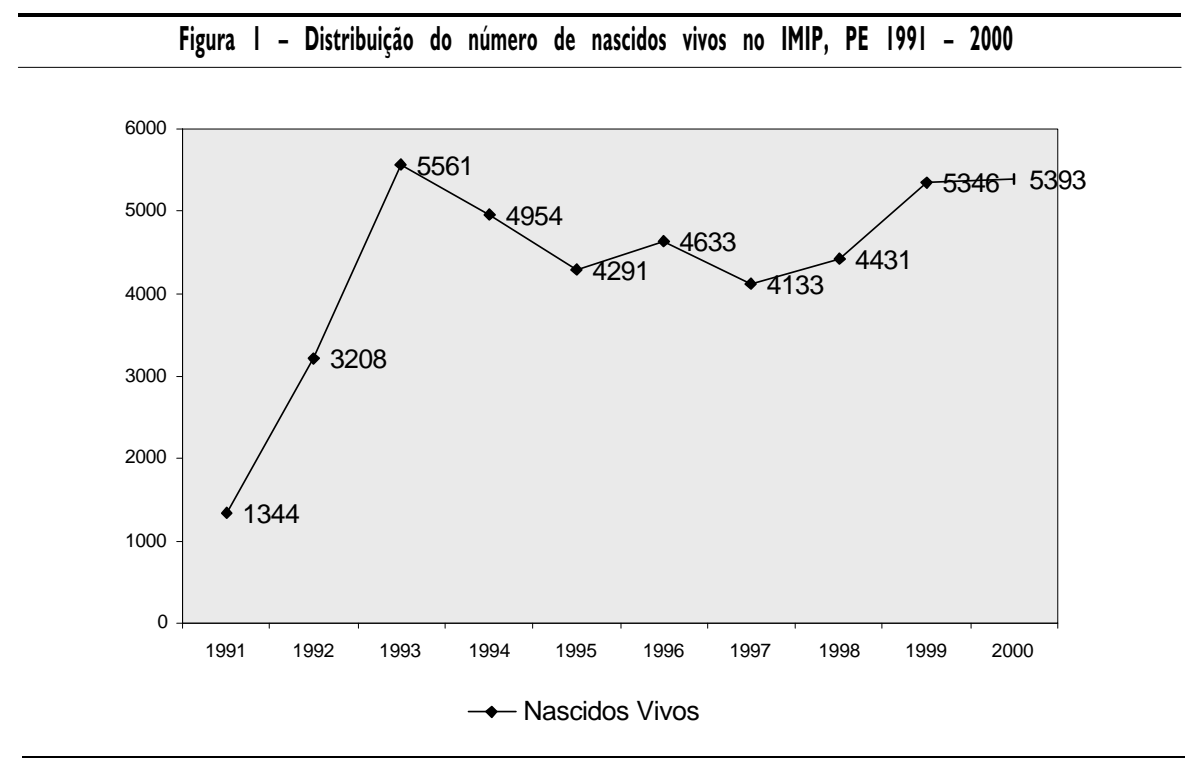

Fonte: SINASC, IMIP e DIEVES/SMS Recife

Declarações de Nascidos Vivos na maternidade do IMIP, no período de julho de 199| a dezembro de 2000.

Utilizou-se o banco de dados secundário do Sinasc obtido no IMIP para o período | 99 |1992, por ser o único existente no estado (piloto) e para os demais anos da série recorreu-se ao setor de Epidemiologia da Secretaria de Saúde da Prefeitura do Recife, em virtude de seus dados serem submetidos à crítica e correção deste o início da implantação.

Para análise estatística foi realizado o Qui-quadrado de tendência com nível de significância estatística $p<0,05$, considerando os anos de 1993 a 2000, por ser os dois primeiros fase inicial de implantação, no programa Epi Info, versão 6.0.

Dentro do elenco de variáveis contidas na DN foram incluídas para a análise aquelas mais representativas na adoção de medidas de vigilância para a redução da mortalidade infantil, como as condições da criança ao nascimento: peso ao nascer e índice de Apgar; sobre a gestação e o parto: duração da gestação, tipo de parto e número de consultas de pré-natal, excluindo-se o tipo de gravidez, por ser insignificante o número de gravidezes múltiplas e as referentes a alguns atributos maternos: idade, escolaridade e número de filhos tidos.

\section{Resultados}

O numero de nascidos vivos no IMIP no período estudado foi 42.994, conforme distribuição exibida na Figura I. Observa-se que houve um aumento no número de nascidos vivos entre o primeiro e o último ano, sendo bem mais evidente entre os anos de 1991 e 1992, quando este acréscimo foi de aproximadamente $100 \%$, isso porque em $199 \mid$ constavam apenas os nascidos vivos referentes ao segundo semestre.

As Tabelas de I a 5 apresentam as variáveis analisadas no estudo, distribuídas conforme informações biológicas e demográficas do recém-nascido e da mãe.

A primeira tabela mostra que até 1997 a maioria dos recém-nascidos pesava mais que $2.500 \mathrm{~g}$, correspondendo em média a $82 \%$ do total. No entanto, o percentual de baixo peso ao nascer aumentou gradativamente ao longo dos anos, principalmente a partir de 1997, quando o valor encontrado foi de $18,3 \%$, atingindo um patamar de 22,8\% em 2000, como comprova a análise de tendência com resultado estatisticamente significante $\chi^{2}$ de tendência $=170,6 ; p<0,01)$. Apenas 30 bebês da série não foram pesados, representando $0,07 \%$ do total, tendo se concentrado nos anos de 1999 e 2000, não se encontrando razão para a omissão da informação.

Com relação ao índice de Apgar, a grande maioria, aproximadamente $80 \%$, apresentou boas condições de vitalidade no primeiro minuto de vida (Apgar entre 8 e 10). Entre as crianças nascidas com hipóxia grave (Apgar de 0 a 3 ), os percentuais situaram-se entre 3,9\% em 1993 e 5,2\% em 2000. Para o Apgar no 5o minuto, foram encontrados mais de $90 \%$ 


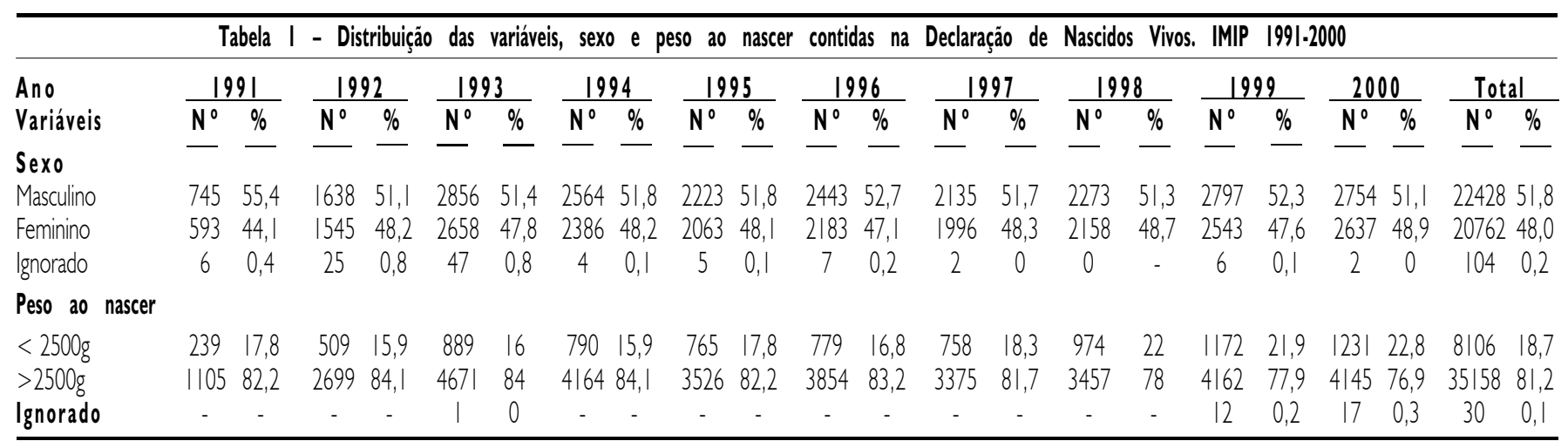

Fonte: SINASC, IMIP e DIEVESSMS Recife

\begin{tabular}{|c|c|c|c|c|c|c|c|c|c|c|c|c|c|c|c|c|c|c|c|c|c|c|}
\hline \multirow{3}{*}{$\begin{array}{l}\text { Ano } \\
\text { Variáveis } \\
\text { Apgar } 1^{\circ} \text { minuto }\end{array}$} & \multicolumn{2}{|c|}{1991} & \multicolumn{2}{|c|}{1992} & \multicolumn{2}{|c|}{1993} & \multicolumn{2}{|c|}{1994} & \multicolumn{2}{|c|}{1995} & \multicolumn{2}{|c|}{1996} & \multicolumn{2}{|c|}{1997} & \multicolumn{2}{|c|}{1998} & \multicolumn{2}{|c|}{1999} & \multicolumn{2}{|c|}{2000} & \multicolumn{2}{|c|}{ Total } \\
\hline & $\mathrm{N}^{0}$ & $\%$ & $\mathrm{~N}^{0}$ & $\%$ & $\mathrm{~N}^{0}$ & $\%$ & $\mathrm{~N}^{0}$ & $\%$ & $\mathrm{~N}^{0}$ & $\%$ & $\mathrm{~N}^{0}$ & $\%$ & $\mathrm{~N}^{0}$ & $\%$ & $\mathrm{~N}^{0}$ & $\%$ & $\overline{N^{0}}$ & $\%$ & $\mathrm{~N}^{0}$ & $\%$ & $\mathrm{~N}^{0}$ & $\%$ \\
\hline & & & & & & - & & & 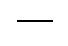 & 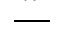 & - & 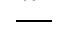 & - & - & - & - & - & & - & 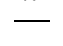 & - & 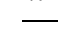 \\
\hline $0 a 3$ & 74 & 5,5 & 110 & 3,4 & 217 & 3,9 & 207 & 4,2 & 221 & 5,2 & 194 & 4,2 & 168 & 4,1 & 205 & 4,6 & 251 & 4,7 & 278 & 5,2 & 1925 & 4,5 \\
\hline $4 a 7$ & 193 & 14,4 & 379 & 11,8 & 715 & 12,9 & 661 & 13,3 & 638 & 14,9 & 641 & 13,8 & 626 & $|5|$, & 666 & 15 & 823 & 15,4 & 949 & 17,6 & 6291 & 14,5 \\
\hline 8 a 10 & $|05|$ & 78,2 & 2656 & 82,8 & 4525 & 81,4 & 4049 & 81,7 & 3384 & 78,9 & 3777 & 81,5 & 3319 & 80,3 & 3528 & 79,6 & 4206 & 78,7 & 4097 & 76 & 34592 & 79,9 \\
\hline 0 a3 & 18 & 1,3 & 24 & 0,7 & 40 & 0,7 & 58 & 1,2 & 54 & 1,3 & 58 & 1,3 & 47 & $||$, & 37 & 0,8 & 73 & 1,4 & 83 & 1,6 & 492 & 1,1 \\
\hline $4 a 7$ & 68 & 5,1 & 122 & 3,8 & 227 & 4,1 & 213 & 4,3 & 261 & 6,1 & 199 & 4,3 & 182 & 4,4 & 216 & 4,9 & 222 & 4,2 & 298 & 5,5 & 2008 & 4,6 \\
\hline 8 a 10 & $|24|$ & 92,3 & 3009 & 93,8 & 5218 & 93,8 & 4668 & 94,2 & 3957 & 92,2 & 4367 & 94,3 & 3899 & 94,3 & 4170 & 94,1 & 5042 & 94,3 & 4983 & 92,4 & 40554 & 93,7 \\
\hline Ignorado & 17 & 1,3 & 53 & 1,7 & 76 & 1,4 & 15 & 0,3 & 19 & 0,4 & 9 & 0,2 & 5 & 0,1 & 8 & 0,2 & 9 & 0,2 & 29 & 0,5 & 240 & 0,6 \\
\hline
\end{tabular}

Fonte: SINASC, IMIP e DIEVES/SMS Recife

Tabela 3 - Distribuição variáveis idade gestacional, tipo de parto e consultas de pré-natal contidas na Declaração de Nascidos Vivos. IMIP 1991 - 2000

\begin{tabular}{|c|c|c|c|c|c|c|c|c|c|c|c|c|c|c|c|c|c|c|c|c|c|c|}
\hline \multirow{2}{*}{$\begin{array}{l}\text { Ano } \\
\text { Variáveis }\end{array}$} & \multicolumn{2}{|c|}{1991} & \multicolumn{2}{|c|}{1992} & \multicolumn{2}{|c|}{1993} & \multicolumn{2}{|c|}{1994} & \multicolumn{2}{|c|}{1995} & \multicolumn{2}{|c|}{1996} & \multicolumn{2}{|c|}{1997} & \multicolumn{2}{|c|}{1998} & \multicolumn{2}{|c|}{1999} & \multicolumn{2}{|c|}{2000} & \multicolumn{2}{|c|}{ Total } \\
\hline & $\mathrm{N}^{0}$ & $\%$ & $\mathrm{~N}^{0}$ & $\%$ & $\mathrm{~N}^{0}$ & $\%$ & $\mathrm{~N}^{0}$ & $\%$ & $\mathrm{~N}^{0}$ & $\%$ & $\mathrm{~N}^{0}$ & $\%$ & $\mathrm{~N}^{0}$ & $\%$ & $\mathrm{~N}^{0}$ & $\%$ & $\mathrm{~N}^{0}$ & $\%$ & $\mathrm{~N}^{0}$ & $\%$ & $\mathrm{~N}^{0}$ & $\%$ \\
\hline $\begin{array}{l}\text { Idade gesta } \\
>37 \text { semanas } \\
<37 \text { semanas } \\
\text { |gnorado }\end{array}$ & $\begin{array}{c}1280 \\
36 \\
28\end{array}$ & $\begin{array}{l}95,2 \\
2,7 \\
2,1\end{array}$ & $\begin{array}{c}3123 \\
20 \\
65\end{array}$ & $\begin{array}{c}97,4 \\
0,6 \\
2\end{array}$ & $\begin{array}{c}4775 \\
542 \\
244\end{array}$ & $\begin{array}{c}85,9 \\
9,7 \\
4,4\end{array}$ & $\begin{array}{c}4274 \\
646 \\
34\end{array}$ & $\begin{array}{c}86,3 \\
13 \\
0,7\end{array}$ & $\begin{array}{c}3562 \\
726 \\
3\end{array}$ & $\begin{array}{c}83 \\
16,9 \\
0,1\end{array}$ & $\begin{array}{c}3890 \\
742 \\
1\end{array}$ & $\begin{array}{l}84 \\
16 \\
-\end{array}$ & $\begin{array}{c}3410 \\
714 \\
9\end{array}$ & $\begin{array}{c}82,5 \\
17,3 \\
0,2\end{array}$ & $\begin{array}{c}3500 \\
916 \\
15\end{array}$ & $\begin{array}{c}79,1 \\
20,6 \\
0,3\end{array}$ & $\begin{array}{c}4166 \\
1176 \\
4\end{array}$ & $\begin{array}{l}77,9 \\
22 \\
0,1\end{array}$ & $\begin{array}{c}410 \mid \\
1284 \\
8\end{array}$ & $\begin{array}{c}76,1 \\
23,8 \\
0,1\end{array}$ & $\begin{array}{c}36081 \\
6802 \\
411\end{array}$ & $\begin{array}{r}83,3 \\
15,7 \\
1,0\end{array}$ \\
\hline $\begin{array}{l}\text { Tipo de part } \\
\text { Normal } \\
\text { Cesáreo } \\
\text { Ignorado }\end{array}$ & $\begin{array}{c}837 \\
502 \\
5\end{array}$ & $\begin{array}{c}62,3 \\
37,4 \\
0,4\end{array}$ & $\begin{array}{c}2160 \\
1039 \\
9\end{array}$ & $\begin{array}{c}67,3 \\
32,4 \\
0,3\end{array}$ & $\begin{array}{c}3868 \\
1635 \\
58\end{array}$ & $\begin{array}{c}69,6 \\
29,4 \\
1\end{array}$ & $\begin{array}{c}3534 \\
14 \mid 6 \\
4\end{array}$ & $\begin{array}{c}71,3 \\
28,6 \\
0,1\end{array}$ & $\begin{array}{c}2697 \\
|59| \\
3\end{array}$ & $\begin{array}{c}62,9 \\
37,1 \\
0,1\end{array}$ & $\begin{array}{c}2926 \\
1706 \\
1\end{array}$ & $\begin{array}{c}63,2 \\
36,8 \\
0\end{array}$ & $\begin{array}{c}265 \mid \\
|48| \\
\mid\end{array}$ & $\begin{array}{c}64,1 \\
35,8 \\
0\end{array}$ & $\begin{array}{c}2797 \\
1633 \\
1\end{array}$ & $\begin{array}{c}63,1 \\
36,9 \\
0\end{array}$ & $\begin{array}{c}3438 \\
1904 \\
4\end{array}$ & $\begin{array}{c}64,3 \\
35,6 \\
0,1\end{array}$ & $\begin{array}{c}3490 \\
1900 \\
3\end{array}$ & $\begin{array}{c}64,7 \\
35,2 \\
0,1\end{array}$ & $\begin{array}{c}28398 \\
14807 \\
89\end{array}$ & $\begin{array}{l}65,6 \\
34,2 \\
0,2\end{array}$ \\
\hline $\begin{array}{l}\text { Consultas de } \\
\text { Nenhuma } \\
\text { I a } 6 \\
7 \text { e mais } \\
\text { Ignorado }\end{array}$ & natal & & & & & & & & $\begin{array}{c}208 \\
1271 \\
576 \\
2236\end{array}$ & $\begin{array}{l}4,8 \\
29,6 \\
13,4 \\
52,1\end{array}$ & $\begin{array}{c}331 \\
2803 \\
1367 \\
132\end{array}$ & $\begin{array}{c}7,1 \\
60,5 \\
29,5 \\
2,8\end{array}$ & $\begin{array}{c}313 \\
2499 \\
1251 \\
70\end{array}$ & $\begin{array}{c}7,6 \\
60,5 \\
30,3 \\
1,7\end{array}$ & $\begin{array}{l}315 \\
2513 \\
1437 \\
166\end{array}$ & $\begin{array}{c}7,1 \\
56,7 \\
32,4 \\
3,7\end{array}$ & $\begin{array}{c}35 \mid \\
858 \\
1540 \\
160\end{array}$ & $\begin{array}{c}6,6 \\
61,6 \\
28,8 \\
3\end{array}$ & $\begin{array}{c}326 \\
3274 \\
1654 \\
139\end{array}$ & $\begin{array}{c}6 \\
60,7 \\
30,7 \\
2,6\end{array}$ & $\begin{array}{l}1844 \\
13218 \\
7825 \\
2903\end{array}$ & $\begin{array}{l}7,1 \\
51,3 \\
30,3 \\
11,3\end{array}$ \\
\hline
\end{tabular}

Fonte: SINASC, IMIP e DIEVES/SMS Recife

de bebês em boas condições de vitalidade e, na faixa de 0 a 3 , a proporção máxima observada foi de I,6\% em 2000 e a mínima de 0,7\% em 1992 (Tabela 2).

No tocante à variável idade gestacional (Tabela 3), verificou-se que o percentual de recém-nascidos pré-termo (menos de 37 semanas) sofreu incremento progressivo, ao longo dos anos, variando de 9,7\% em 1993 a 23,8\% em $2000\left(\chi^{2}\right.$ de tendência $=503,6$; $\mathrm{p}<0,0 \mathrm{l})$.

Ainda na Tabela 3, os resultados referentes ao tipo de parto mostram que a maioria foi vaginal, representando em média $65,8 \%$ ao longo da década. No entanto, verificou-se uma elevada taxa de cesárea, variando de 29,4\% em 1993 a 35,2\% em 2000, mantendo tendência à progressão, como se observa na análise estatística $\left(\chi^{2}\right.$ de tendência $=73,7 ; p<$ $0,00 \mathrm{I}$ ). Quanto à variável número de consultas de pré-natal, introduzida na DN só a partir de 


\begin{tabular}{|c|c|c|c|c|c|c|c|c|c|c|c|c|c|c|c|c|c|c|c|c|c|c|}
\hline \multirow{3}{*}{$\begin{array}{l}\text { Ano } \\
\text { Variáveis }\end{array}$} & \multicolumn{22}{|c|}{ Tabela 4 - Distribuição das variáveis maternas - idade e escolaridade contidas na Declaração de Nascidos Vivos. IMIP $\quad$ 1991-2000 } \\
\hline & \multicolumn{2}{|c|}{1991} & \multicolumn{2}{|c|}{1992} & \multicolumn{2}{|c|}{1993} & \multicolumn{2}{|c|}{1994} & \multicolumn{2}{|c|}{1995} & \multicolumn{2}{|c|}{1996} & \multicolumn{2}{|c|}{1997} & \multicolumn{2}{|c|}{1998} & \multicolumn{2}{|c|}{1999} & \multicolumn{2}{|c|}{2000} & \multicolumn{2}{|c|}{ Total } \\
\hline & $\mathrm{N}^{0}$ & $\%$ & $\mathrm{~N}^{0}$ & $\%$ & $\mathrm{~N}^{0}$ & $\%$ & $\mathrm{~N}^{0}$ & $\%$ & $\mathrm{~N}^{0}$ & $\%$ & $\mathrm{~N}^{0}$ & $\%$ & $\mathrm{~N}^{0}$ & $\%$ & $\mathrm{~N}^{0}$ & $\%$ & $\mathrm{~N}^{0}$ & $\%$ & $\mathrm{~N}^{0}$ & $\%$ & $\mathrm{~N}^{0}$ & $\%$ \\
\hline Idade materna & & & & & & & & & & & & & & & & & & & & & & \\
\hline$<20$ & 334 & 24,9 & 822 & 25,6 & 1340 & 24,1 & 1342 & 27,1 & $|20|$ & 28 & 1306 & 628,2 & 1488 & 36 & 1282 & 28,9 & 1558 & 29,1 & 1552 & 28,8 & 12225 & 28,2 \\
\hline 20 a 34 & & 64,7 & 1938 & 60,4 & 3353 & 60,3 & 3284 & 66,3 & 2865 & 66,8 & 82 & 66 & 2390 & 57,8 & 2892 & 65,3 & 3437 & 64,3 & 3484 & 64,6 & 27594 & +6 \\
\hline 5 e mais & 80 & 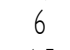 & 183 & 5,7 & $27 \mid$ & 4,9 & 261 & 5,3 & 210 & 4,9 & 228 & 4,9 & 230 & 5,6 & 235 & 5,3 & 346 & 6,5 & 337 & 6,2 & 2381 & 5,5 \\
\hline norado & 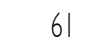 & 4,5 & 265 & 8,3 & 597 & 10,7 & 67 & 1,4 & 15 & 0,3 & 17 & 0 & 25 & 0,6 & 22 & 0,5 & 5 & 0,1 & 20 & 0,4 & 1094 & 5 \\
\hline Escolaridade & materna & & & & & & & & & & & & & & & & & & & & & \\
\hline Nenhuma & & & & & 310 & 5,6 & 248 & 5 & 242 & 5,6 & 225 & 4,9 & 147 & 3,6 & 165 & 3,7 & 172 & 3,2 & |7| & 3,2 & 1680 & 4,5 \\
\hline 3 & & & & & 2963 & 53,3 & 2823 & 57 & 2534 & 59 & 2757 & 759 & 2463 & 59 & 2468 & 55,7 & & & 496 & 97 & & 44 \\
\hline $4 a 7$ & & & & & 976 & 17,6 & 879 & 17,7 & 671 & 15,6 & 717 & 15 & 699 & 16,9 & 734 & 16,6 & 1433 & 26,7 & 2449 & 45,4 & 8558 & 22,7 \\
\hline $8 a l l$ & & & & & 953 & 17,1 & 864 & 17,4 & 759 & 17,7 & 852 & 18,4 & 762 & 18,4 & 978 & 22,1 & 1713 & 32 & 1874 & 34,7 & 8755 & 23,2 \\
\hline $12 e+$ & & & & & 129 & 2,3 & 93 & 1,9 & 60 & 1,4 & 66 & 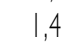 & 48 & 1,2 & 62 & 1,4 & 303 & 5,7 & 312 & 5,8 & 1073 & 2,8 \\
\hline $\operatorname{lgn} /$ Não inf. & & & & & 230 & 4,1 & 47 & 0,9 & 25 & 0,6 & 16 & 0,3 & 14 & 0,3 & 24 & 0,5 & 165 & 3 & 91 & 1,7 & 612 & 1,6 \\
\hline
\end{tabular}

Fonte: SINASC, IMIP e DIEVESSMS Recife

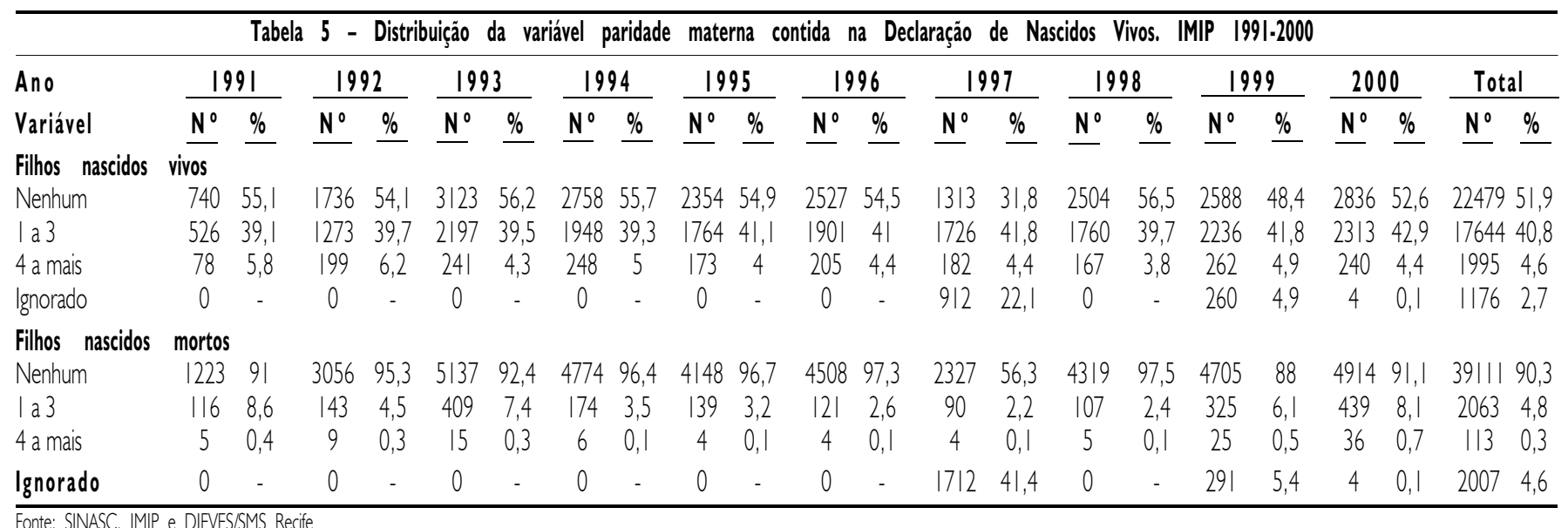

1995. A partir do ano de 1996, percebe-se uma melhora significativa do preenchimento dessa variável, encontrando-se apenas 3\% de ignorados ou sem informação. Excluindo o ano de 1995, nos demais há registro de que cerca de um terço das mães havia realizado sete ou mais consultas de pré-natal.

Em relação à idade materna, houve um aumento proporcional de mulheres com menos de 20 anos, constatado na análise de tendência $\left(\chi^{2}\right.$ de tendência $=13,3 ; p<$ 0,00 I). No tocante à escolaridade materna, os diferentes modelos de formulários de DN dificultaram a análise dos resultados. Só a partir do ano de 1999 foi categorizado em nenhuma, I a 3 anos, 4 a 7, 8 a | I, 12 e mais e ignorado. Com exceção dos itens "nenhuma" e "ignorado/sem informação", presentes em todos os modelos, os valores variaram de 5,6\% a 3,2\% para o primeiro e de $4,1 \%$ a $1,7 \%$ para o segundo, entre os anos de 1993 e 2000, respectivamente (Tabela 4).
Para a variável filhos nascidos vivos ressaltase que, durante toda a década, o maior contingente de recém-nascidos era constituído de primogênitos, sendo o mínimo de 48,4\% (1999) e o máximo de 56,5\% (1998). Quanto aos filhos nascidos mortos a grande maioria das mães (mais de 90\%) não tinha tido nenhum natimorto. Estas duas variáveis apresentaram um padrão atípico, no ano de 1997, quando o percentual encontrado para as categorias citadas foi de $31,8 \%$ para os filhos nascidos vivos e de $56,3 \%$ para os filhos nascidos mortos, com elevado percentual de "ignorados" (Tabela 5).

\section{Discussão}

A análise da série temporal dos nascidos vivos da década de 90, no Instituto Materno Infantil de Pernambuco (IMIP), tem uma importância fundamental para o planejamento institucional de ações voltadas ao grupo populacional neonatal, que é o mais vulnerável ao óbito entre as crianças menores de um ano neste serviço.
Através da análise dos dados obtidos ao longo dos anos 90, verificou-se que, no perfil de nascimentos, predominam crianças nascidas de parto vaginal, com peso igual ou superior a 2500g, a termo (idade gestacional maior que 37 semanas), Apgar no $1^{0}$ e $5^{0}$ minutos entre 8 e 10, filhos de mulheres adultas (20 anos a mais) que realizaram, em sua maioria, 4 a 6 consultas de pré-natal.

Os resultados mostraram que a maioria dos partos foi vaginal, no entanto, encontrou-se um percentual médio de 35\% de cesarianas, elevado quando comparado ao obtido nos Estados Unidos em 1991 (23,5\%), que levou oCenters for Disease Control and Prevention (CDC) a lançar como meta de saúde pública para o ano 2000 a redução dessa taxa para 15\%'. A despeito dos valores encontrados na unidade em foco serem muito altos, explicáveis talvez por ser referência estadual para gravidezes de alto risco, são menores do que os verificados por Silva no Rio de Janeiro, Maia em Minas Gerais e 
Wisbeck em Blumenau (59, I\% a 41,7\%) $)^{5}, 10,11$. No entanto, se aproximam aos descritos por Loja, em $1993^{12}$ e Caetano em 1997'3, analisando o Sinasc do Hospital Pedro Ernesto, no Estado do Rio de Janeiro, que tem características semelhantes ao IMIP e que foram respectivamente $31,6 \%$ e $38,5 \%$.

Apesar da predominância do adequado peso ao nascer, houve um expressivo contingente de crianças nascidas com baixo peso, cujo percentual encontrado foi de $17,8 \%$ em |99|, com um aumento gradual ao longo dos anos, chegando em 2000 a 22,8\%. Este dado é bem maior do que os relatados na maioria dos artigos consultados, baseados em estudos populacionais, cujos valores estavam em torno de 10\%5,10,14. Contudo, num estudo realizado em 1993, no Hospital Pedro Ernesto no Rio de Janeiro, o percentual relatado foi de $15,1 \%$, um pouco mais próximo aos nossos no mesmo ano ${ }^{12}$.

Sabe-se que dentre os vários indicadores de saúde e das condições de vida de uma população destaca-se o peso ao nascer, pela sua importância na predição da morbi-mortalidade infanti $\left.\right|^{15,16}$. $O$ baixo peso ao nascer, presente em gestações a termo e únicas, sugere a presença de intercorrências maternas, como desnutrição, infecções e transtornos hipertensivos, que podem afetar o desenvolvimento intra-uterino, colocando em risco a capacidade de sobrevivência desses recém-nascidos ${ }^{5}$.

Alguns fatores relacionados ao baixo peso ao nascer estão presentes na DN, como: a idade materna nos extremos da vida reprodutiva ${ }^{17}$, representada em nosso estudo por percentuais que variaram de $24,1 \%$ a $28,8 \%$ para mães adolescentes; a pouca escolaridade e a grande paridade, reflexo da baixa condição socioeconômica. A gravidez na adolescência (faixa etária de 10 a 19 anos) compreende uma série de condições anatômicas, fisiológicas, psicológicas, sociais e culturais que podem determinar um maior risco para a mortalidade perinatal e materna. Este grupo representou uma proporção maior que os descritos na literatura consultada, Maia $^{10}$ (19,5\%) e Loja ${ }^{12}$ (I8\%), possivelmente por se tratar de um hospital de referência estadual.

Tanto o baixo peso ao nascer quanto a prematuridade (idade gestacional menor que 37 semanas) evidenciam problemas no desenvolvimento intra-uterino. Foi observado um percentual de prematuridade progressivamente maior ao longo dos anos analisados, variando de $9,7 \%$ em 1993 para 23,8\% em 2000, bem mais elevados que os observados por Silva $(6,4 \%)^{5}$, Costa \& Gotlieb $(7,6 \%)^{17}$. Percentuais próximos a esses foram encontrados ao se estudar os nascidos vivos residentes no município do Recife no período de 1995 a 2000, que variou de 9,2\% a 8,5\%. ${ }^{9}$ Essas discrepâncias talvez se justifiquem pelo fato dos autores citados analisarem os nascidos vivos residentes no município em contraste com a ocorrência de uma unidade hospitalar com nível de complexidade terciário.

O índice de Apgar traduz as condições de vitalidade do recém-nascido; é uma medida de fácil obtenção sendo importante para identificação de bebês de risco. Apesar de ser um bom indicador das condições clínicas do neonato, freqüentemente esta variável não é preenchida nas Declarações de Nascidos Vivos, inviabilizando sua análise ${ }^{1,5}$. Sua mensuração só é confiável nos serviços que dispõem de neonatologistas em todos os partos, como é o caso da unidade de saúde em estudo, onde foram encontrados valores que variaram de 3,4\% a 5,5\% para Índice de Apgar entre 0 e 3 no 10 minuto e de $0,7 \%$ a $1,6 \%$ no $5^{\circ}$ minuto, percentuais elevados quando comparados aos encontrados pelos autores acima citados $(2,3 \% \text { em ambos })^{1,5}$.

Quanto ao número de gestantes que realizaram consulta pré-natal, cerca de um terço das mães dos nascidos vivos havia realizado mais de sete consultas e $45 \%$, quatro a seis, nos dois últimos anos ( 1999 e 2000), dados muito próximos aos descritos pela pesquisa nacional em 1996, realizada pela Bemfam ${ }^{18}$ cuja freqüência de realização de quatro ou mais consultas de pré-natal foi da ordem de $77 \%$. Segundo o Programa de Assistência Integral à Saúde da Mulher Paism - a gestante é considerada assistida no pré-natal quando comparece a um número mínimo de seis consultas durante a gravidez ${ }^{19}$. Aparentemente, esses achados são animadores, porém sua interpretação merece cautela pois os resultados apresentam um importante problema metodológico. Como na categorização desta variável no formulário da DN foram agregadas as mulheres que realizaram quatro e cinco consultas às que compareceram a seis, este fato inviabilizou a comparabilidade das informações aqui obtidas com outros estudos onde a mesma apresenta diferentes pontos de corte, permitindo desagregação dos dados.

Através da análise da série histórica do Sinasc no IMIP, identificou-se uma elevada taxa de cesariana (35\%), que somada ao expressivo percentual de baixo peso ao nascer (22,8\%), de prematuridade $(23,8 \%)$, proporção de Apgar no $I^{\circ}$ minuto $<4(5,2 \%)$, ainda associada a um alto contingente de mães adolescentes (28,8\%), mostrou situações de perigo à sobrevivência infantil, sendo possível diferenciar e orientar as necessidades de vigilância e monitoramento nos serviços de puericultura para o grupo de maior risco.

\section{Conflito de interesse: não há.}

\section{SUMmary}

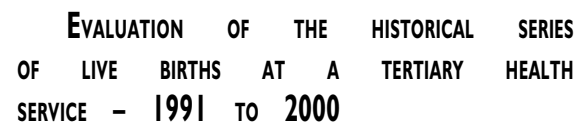

O BJECTNES. Thisstudy aimed to analyze some variables fo und in the Birth Certificates at the Instituto Materno Infantil de Pernambuco, Recife, from July 199 I to December 2000, according to Sinasc (National Information's data base of Births).

Methods. The statistical analysis was performed using the chi-square test for trend $(p<0.05)$ on Epilnfo software (version 6.0), considering the years 1993 to 2000.

REsults. The sample comprised a predominance of non-surgical deliveries, adequate gestational age of newborns, weight $\geq 2500 \mathrm{~g}$, Apgarscore $1{ }^{\text {st }}$ min and $5^{\text {th }}$ min between $8-10$ and mature mothers who had attended from 4 to 6 appo intments at prenatal care system. Itshowed the following rates: cesarean-section of $29.4 \%$ to $35.2 \%(? 2=73.7 ; p<0.01)$, low weight at birth of $15.9 \%$ to $22.8 \%$ (?2=170.6; $p<0.01)$; premature newborns of $9.7 \%$ to $23.8 \%$ (?2= 503.6; $p<0.01$ ); teenage mothers of $24.1 \%$ to $28.8 \%(? 2=13.3 ; p<0.01)$, moreover a high percentage of newborns with Apgarindex lower than 3 at the I $^{\text {st minute }}(3.4 \%$ to $5 . \%)$ and $(0.7 \%$ to $1.6 \%)$ at the $5^{\text {th }}$ minute.

Conclusions. These results confirm the role of IMIP as an institution for referral of high-risk pregnancies in Pernambuco. [Rev Assoc Med Bras 2005; 5 I (I): 17-22]

KEY WORDS: Health assessment. Information systems. Birth certificates. 


\section{ReferênCIAS}

I. Mello Jorge MHP, Gotlieb SLD, Soboll MLMS, Almeida MF, Latorre MRDO. Avaliação do sistema de informação sobre nascidos vivos e seu uso de dados em epidemiologia e estatísticas de saúde. Rev Saúde Pública 1993;27(Supl 6):3-46.

2. Mello Jorge MHP, Gotlieb SLD, Oliveira H. O sistema de informação sobre nascidos vivos: primeira avaliação dos dados brasileiros. Inf Epidemiol SUS 1996;5: I 5-48.

3. Carvalho DM. Grandes sistemas de informação em saúde: revisão e discussão da situação atual. Inf Epidemiol SUS 1997; 4:7-46.

4. Fernandes DM. Avaliação da qualidade de informaç̧ões do SIM e SINASC: uma experiência na concatenação de informações do sistema de estatísticas vitais Distrito Federal 1989-1991. In: XI Encontro Nacional de Estudos Populacionais da Associação Brasileira de Estudos Populacional, ABEP; 1998. Caxambu. Disponível em: http://www.abep. cedeplar.ufmg.br/docs/anais/pdf/I 998/a. [Acesso em: 200 I]

5. Silva RI, Theme Filha MM, Noronha CP. Sistema de informação sobre nascidos vivos na cidade do Rio de Janeiro 1993/1996. Inf Epidemiol SUS 1997;2:33-48.

6. Ministério da Saúde. Fundação Nacional de Saúde. Portaria n.475 de 31 de agosto de 2000. Republicada com as alterações efetuadas pela Portaria n.627, de 5 de dezembro de 200I. Diário Oficial da União, Brasília (DF) 200।;238, I 4 dez 200।.
7. Mishima FC, Socochi CGS, Ferro MAR, Lima RAG, Costa MAR. Declaração de nascidos vivos: análise do seu preenchimento no município de Ribeirão Preto, São Paulo, Brasil. Cad Saúde Pública 1999; | 5:387-95.

8. Ministério da Saúde. Fundação Nacional de Saúde. Manual de instruções para o preenchimento da declaração de nascido vivo. $3^{\text {a }} \mathrm{ed}$. Brasília (DF): Centro de Documentação do Ministério da Saúde; 200 I

9. Recife. Secretaria de Saúde. Crianças do Recife: perfil de nascimentos, 1995-2000. Recife: Secretaria de Saúde; 2002.

10. Maia MAC. Caracterização dos nascidos vivos hospitalares no primeiro ano de implantação do sub-sistema sobre nascidos vivos, em município de Minas Gerais, Brasil, 1996. Rev Saúde Pública 1997;31:58|-5.

I I. Wisbeck J, SantaHelena ET. Implantação do Sinasc e perfil dos nascidos vivos em Blumenau, 1994-1997. In: IV Congresso Brasileiro de Epidemiologia; 1998; Rio de Janeiro. Resumos. Rio de Janeiro: Associação Brasileira de Pós-Graduação em Saúde Coletiva (Abrasco); 1998. p.166, resumo 97

12. Loja T, Lima CXB, Caetano R, Vater C, Nogueira MC. Implantação do sistema de nascidos vivos no Hospital Universitário Pedro Ernesto - UERJ. In: $V$ Congresso Brasileiro de Saúde Coletiva; V Congresso Paulista de Saúde Pública; 1997; Águas de Lindóia. Resumos. São Paulo: Associação Brasileira de Pós-Graduação em Saúde Coletiva (Abrasco); 1997. p.221, resumo 412 .
13. Caetano R, Loja TB, Lima CXB. Perfil dos nascidos vivos de 1997 no Hospital Universitário Pedro Ernesto - UERJ. In: IV Congresso Brasileiro de Epidemiologia; 1998; Rio de Janeiro. Resumos. Rio de Janeiro: Associação Brasileira de PósGraduação em Saúde Coletiva (Abrasco); 1998. p.166, resumo 96.

14. Guimarães MJB. Mortalidade infantil e condições de vida: uma análise da desigualdade espacial no Recife [dissertação]. Recife: Instituto Materno Infantil de Pernambuco (IMIP); 1998.

15. Puffer RR, Serrano CV. Distribuición del peso ao nascer. Washington (DC): Organización Panamericana de la Salud (OPS); 1988. (Publicación Cientifica, n.504).

16. Victora CG, Barros FC. Epidemiologia da desigualdade. $2^{\text {a }}$ ed. São Paulo: Hucitec; 1989.

17. Costa CE, Gottlieb SLD. Estudo epidemiológico do peso ao nascer a partir da Declaração de Nascidos Vivo. Rev Saúde Pública 1998; 32:328-34.

18. BEMFAM (Sociedade Civil Bem-Estar Familiar no Brasil). Pesquisa Nacional sobre Demografia e Saúde: 1996. $2^{\mathrm{a}}$ ed. Rio de Janeiro: Bemfam; 1996.

19. Ministério da Saúde. Acompanhamento à saúde da mulher. Parte I: gestação, parto e puerpério. Brasília (DF): Ministério da Saúde; 1994.

Artigo recebido: 04/I I/2003

Aceito para publicação: 15/04/2004

\title{
www.ramb.org.br
}

\author{
A Ramb disponibiliza agora um novo serviço para os nossos colaboradores: \\ 0 envio de artigos pela internet. Através do nosso publicador, que agiliza o fluxo \\ de submissão com mais rapidez e versatilidade, você poderá enviar seu artigo \\ para publicação na Ramb. Experimente: é muito mais cômodo, muito mais rápido e \\ você ainda poderá acompanhar on line o curso de sua apreciação. \\ Acesse www.ramb.org.br
}

